\title{
Braquiterapia con hilos extraesclerales de iridio-192 en melanomas uveales posteriores. Resultados a largo plazo
}

\author{
S. Valverde Almohalla', B. Díaz Vega², J. M. Tomás San Pedro³ , J. L. Encinas Martín ${ }^{4}$
}

\section{Resumen}

- Propósito: evaluar la eficacia y la seguridad a largo plazo de la braquiterapia con hilos extraesclerales de iridio-192 en melanoma uveal posterior.

- Material y métodos: serie retrospectiva no controlada de 241 pacientes tratados entre enero de 1983 y junio de 2005 en un hospital de referencia nacional.

- Resultados: seguimiento medio de 5,4 años, con $17 \%$ de pérdidas de seguimiento, probabilidades de supervivencia libre de enfermedad a 5 y 10 años del 77\% (I.C. del 95\%: 72; 83) y del 64\% (I.C. del 95\%: 56; 73), de conservar el ojo a 5 y 10 años del 74\% (I.C. del $95 \%$ : 68; 80) y del 63\% (I.C. del 95\%: 55; 71), de conservan una agudeza visual de al menos 0,05 a 1, 5 y 10 años del 64\% (I.C. del 95\%: 57; 71), del 28\% (I.C. del 95\%: $21 ; 35$ ) y del 15\% (I.C. del 95\%: 8; 23). La probabilidad a 5 años de enucleación por complicaciones fue del 11\% (I.C. del 95\%: 6; 16) en melanomas de coroides y del 58\% (I.C. del 95\%: 27; 89) en melanomas de cuerpo ciliar $(\mathrm{p}<0.001)$.

- Conclusiones: la braquiterapia con hilos de iridio-192 consigue un control de la enfermedad similar a las placas de iodo-125; sin embargo, la incidencia de enucleación por complicaciones de la radiación es aparentemente mayor en la técnica con iridio-192, especialmente en melanomas de cuerpo ciliar.

\section{Palabras clave:}

Neoplasias uveales. Melanoma. Braquiterapia. Radioisótopos de iridio. Probabilidad de supervivencia. Supervivencia libre de enfermedad

Oncología, 2005; 28 (9):415-425

\footnotetext{
${ }^{1}$ Médico Especialista en Oftalmología. Médico Adjunto del Servicio de Oftalmología

${ }^{2}$ Médico Especialista en Oftalmología. Médico Adjunto del Servicio de Oftalmología

${ }^{3}$ Médico Interno Residente del Servicio de Oftalmología

${ }^{4}$ Médico Especialista en Oftalmología. Jefe de Servicio de Oftalmología

Hospital Universitario Puerta de Hierro

Madrid
} 


\section{Summary}

- Purpose: To assess the long-term efficay and safeness of iridium-192 wires brachytherapy in posterior uveal melanoma.

- Material and methods: Non-controlled retrospective series of 241 patients, treated from January 1983 to July 2005 in a national referral hospital.

- Results: The mean follow-up was of 5.4 years, with $17 \%$ of the patients lost for follow-up. The 5-year and 10-year probability of disease-free survival was 77\% (95\% IC: 56; 73) and 64\% (95\% IC: 56; 73); and of eye conservation, 74\% (95\% IC: 68; 80) and 63\% (95\% IC: 55; 71). The 1-year, 5-year and 10-year probability of visual acuity conservation of at least 20/400 was 64\% (95\% IC: $57 ; 71$ ), $28 \%$ (95\% IC: $21 ; 35$ ) and $15 \%$ (95\% IC: $8 ; 23$ ), respectively. The 5-year probability of enucleation due to complications was 11\% (95\% IC: 6; 16) for choroidal melanomas, and 58\% (95\% IC: $27 ; 89)$ for ciliary body melanomas ( $\mathrm{p}<0.001)$.

- Conclusion: Iridium-192 wire brachytherapy achieves a disease control similar to that obtained with iodine-125 plaques, but the rate of complications requiring a secondary enucleation seems to be greater with the iridium-192 procedure, especially in ciliary body melanomas.

Key words: Uveal neoplasms. Melanoma. Brachytherapy. Iridium radioisotope. Survival rate. Disease-free survival.

\section{Introducción}

El melanoma uveal es el tumor maligno primario intraocular más frecuente en el adulto. Las medias de edad al diagnóstico están en la sexta década de la vida. Su incidencia en Estados Unidos y Europa es de 5 a 7,5 por millón y año ${ }^{1,2}$. Los melanomas uveales suelen clasificarse topográficamente en melanomas uveales anteriores (melanomas de iris) y melanomas uveales posteriores (M.U.P.). El tratamiento más común de los melanomas de iris es la iridectomía o la iridociclectomía. Los M.U.P. incluyen los melanomas de coroides y los melanomas de cuerpo ciliar. Muchos melanomas de cuerpo ciliar son melanomas ciliocoroideos. El criterio más aceptado en la elección de tratamiento en los M.U.P. es el tamaño. En esencia, los M.U.P. grandes (de más de 8 ó $10 \mathrm{~mm}$. de espesor o de más de $15 \mathrm{~mm}$ de base) se tratan con enucleación del globo ocular ${ }^{3}$, los M.U.P. medianos (desde 2,5 ó $3 \mathrm{~mm}$ de espesor) se tratan con braquiterapia ${ }^{4}$ y los M.U.P. pequeños se vigilan o se tratan con braquiterapia o con termoterapia transpupilar teniendo en cuenta factores clínicos y oftalmoscópicos que predicen riesgo de crecimiento o de metástasis ${ }^{5}$, ${ }^{6}$. El diagnóstico de los M.U.P. suele ser un diagnóstico clínico, pues la especificidad de la oftalmosco- pía por expertos y de la ecografía ocular es conjuntamente de en torno al 99\% , y los procedimientos de diagnóstico citológico, como la punción-aspiración tienen probablemente un balance desfavorable entre sensibilidad, especificidad y riesgos, y se utilizan sólo excepcionalmente. La braquiterapia, o radioterapia con placas o hilos radioactivos extraesclerales, se ha consolidado en la práctica clínica durante las últimas dos décadas como el tratamiento de primera elección de los M.U.P. de tamaño mediano, destacándose sobre otras modalidades menos empleadas de tratamiento conservador como la radioterapia externa con protones o con iones de helio o la resección local (esclerouveectomía parcial lamelar). Durante un siglo, desde las primeras descripciones de esta enfermedad, el diagnóstico de melanoma de coroides estuvo ligado la enucleación del globo ocular, siguiendo el principio de la cirugía oncológica de intentar extirpar por completo el tumor primario ${ }^{8}$. En 1978, Lorenz E. Zimmerman et al., basándose en estudios epidemiológicos retrospectivos, encontraron un incremento de la mortalidad post-enucleación al segundo año seguido de una estabilización al nivel previo a la intervención y lo atribuyeron a diseminación masiva de células tumorales o a una reducción de los mecanismos de defensa inmunológica causa- 
dos por la cirugía9 . Esta hipótesis impulsó el desarrollo de los tratamientos conservadores, sobre todos la braquiterapia y no ha dejado de generar controversias $^{10,11}$ hasta la conclusión del primer ensayo clínico multicéntrico, prospectivo y aleatorizado, el Colaborative Ocular Melanoma Study (C.O.M.S.). Los resultados sobre mortalidad de la parte del C.O.M.S. sobre melanomas coroideos medianos, que comparó la enucleación y la braquiterapia con placas de yodo125 , entre 1985 y 2000 , han demostrado que las tasas de mortalidad eran equivalentes entre ambos tratamientos, confirmando la braquiterapia como tratamiento de elección en melanoma de coroides mediano ${ }^{12-14}$. El objetivo principal de la braquiterapia es reducir el riesgo de metástasis. Sus objetivos secundarios son la conservación del globo ocular y la conservación de alguna función visual en el ojo afectado, aunque la mayoría de los pacientes sufren deterioro severo de la visión en el ojo enfermo a causa de la retinopatía por radiación. Partiendo de una definición minimalista de la retinopatía por radiación, podemos decir que la presentan el $100 \%$ de los ojos tratados. Las complicaciones de la radioterapia en los melanomas de cuerpo ciliar son algo diferentes a las de melanoma de coroides, con mayor incidencia de las de segmento anterior como catarata, glaucoma neovascular y necrosis escleral y menor incidencia de las de segmento posterior como maculopatía por radiación y papilopatía por radiación ${ }^{15}$. En los casos en los que el tumor no responde a la braquiterapia el tratamiento habitual es la enucleación secundaria. Determinadas complicaciones que conducen a un ojo ciego y doloroso son otro motivo de enucleación secundaria. Aunque actualmente el iodo-125 es el isótopo más utilizado en la braquiterapia del melanoma de coroides, se han empleado otros como el cobalto60 , el rutenio-106, el iridio-192 y el paladio-103 ${ }^{16}$. En 1983, los Servicios de Oncología Radioterápica y de Oftalmología de la entonces Clínica Puerta de Hierro, en Madrid, desarrollaron una técnica de braquiterapia para tratar los M.U.P. usando hilos de iridio-192 ${ }^{17}$. Desde entonces el Hospital Universitario Puerta de Hierro recibe pacientes diagnosticados de melanoma uveal derivados de distintos Servicios de Salud españoles. En 1994 publicamos un análisis preliminar de los primeros 66 pacientes ${ }^{18}$. El presente artículo es una revisión retrospectiva de los resultados a largo plazo de esta técnica en un total de 241 pacientes tratados en un periodo de 22 años.

\section{Material y métodos}

\section{Criterios de inclusión de los pacientes}

Hemos revisado las historias clínicas de 241 pacientes consecutivos con diagnóstico de M.U.P. tratados en el Hospital Universitario Puerta de Hierro mediante braquiterapia con hilos epiesclerales de iridio192 entre enero de 1983 y junio de 2005. Además de los pacientes con M.U.P. tratados primariamente con braquiterapia, hemos incluido los pacientes que previamente habían sido tratados con termoterapia transpupilar y tras no responder a dicho tratamiento recibieron braquiterapia como tratamiento secundario.

\section{Manejo de los pacientes: evaluación inicial y descripción de la técnica de tratamiento}

Antes del tratamiento se realizó en el Servicio de Oftalmología a todos los pacientes una exploración oftalmológica basica, incluyendo anamnesis, agudeza visual corregida (la mejor agudeza visual posible con ayuda óptica), biomicroscopía, medición de la presión intraocular y oftalmoscopía de ambos ojos, y además retinografías (fotografías del fondo de ojo), y ecografía ocular del ojo afectado. Tras el diagnóstico clínico de M.U.P., a todos los pacientes se les realizó en el Servicio de Oncología Radioterápica un chequeo para detectar enfermedad metastásica incluyendo exploración física, bioquímica hepática y placa de tórax. La detección de metástasis se consideró contraindicación de la braquiterapia. La técnica de braquiterapia con iridio-192 ya la describimos en publicaciones anteriores ${ }^{17,18}$, pero la comentaremos de nuevo aquí por haber desarrollado en estos años algunas modificaciones. El oftalmólogo realiza la ecografía ocular con un ecógrafo oftálmico de $10 \mathrm{MHz}$ y obtiene las dimensiones ecográficas del tumor, espesor y diámetros basales (transversal y longitudinal). Basándose en estos datos, los Servicios de Oncología Radioterápica y de Radiofísica diseñan los implantes. El iridio-192 es un emisor gamma con buena disponibilidad comercial que se adquiere en forma de "hilos" (alambres) de platino iridiado, que pueden cortarse a la longitud deseada y que por su maleabilidad pueden doblarse para adaptarlos a la curvatura de la esclera. No se emplean placas con este radioisótopo porque no escudarían eficazmente la radiación. La longitud de los hilos de iridio-192 se 
obtiene añadiendo $4 \mathrm{~mm}$ al diámetro longitudinal del tumor. Los hilos de iridio-192 se colocan en tubos de nailon de $0.85 \mathrm{~mm}$ de diámetro externo cuyos extremos se sellan con calor. En el extremo anterior del tubo se hace un agujero que sirve para anclarlo a la esclera. El número de hilos de iridio-192 empleados en un implante se obtiene dividiendo el diámetro transversal del tumor por la separación entre hilos, que varía entre 3 y 4,5 mm y añadiendo un hilo más para cerrar el último espacio. La implantación se realiza con anestesia general. Se hace una peritomía conjuntival, disección roma de la cápsula de Tenon en el cuadrante donde se sitúa el tumor y en los cuadrantes adyacentes, colocación de sedas de 3-0 para tracción bajo los músculos rectos adyacentes, exposición y hemostasia de la zona correspondiente de esclera. Por transiluminación transpupilar con un diafanoscopio, se proyecta la sombra del tumor sobre la esclera y se dibuja sobre esta el margen de la sombra con un rotulador quirúrgico. Se suturan uno a uno los tubos conteniendo los hilos de iridio-192, pararelos al eje longitudinal del tumor, de modo que los hilos de los extremos queden situados sobre los márgenes transversales del tumor. Los puntos se dan con sutura no reabsorbible de 5-0 en el espesor medio de la esclera, en el orificio del extremo anterior de cada tubo a $2 \mathrm{~mm}$ por delante de la sombra tumoral y dos puntos más alrededor de cada tubo, uno de estos lo más posterior posible. Cuando la localización del tumor incluye la inserción de un músculo extraocular se practica en esta un ojal y no es necesario desinsertar el músculo, como sucede con las placas. Los tumores muy posteriores se localizan con oftalmoscopía indirecta e indentación escleral o con ecografía ocular intraoperatoria ${ }^{19}$. La extracción de los hilos de iridio-192 es más breve y se realiza con anestesia retrobulbar, pues basta con retirar soló el punto anterior de cada hilo para poder extraerlo. Se hacen radiografías ortogonales postoperatorias para la planificación de la dosis. Para tumores de hasta $5 \mathrm{~mm}$ de espesor se prescriben las dosis de radiación a $5 \mathrm{~mm}$ y para tumores de más de $5 \mathrm{~mm}$ las dosis se prescriben en el apex del tumor.

\section{Seguimiento}

El seguimiento de los pacientes se realizó en los Servicios de Oftalmología y Oncología Radioterápica, inicialmente en intervalos de 3 meses, aumentán- dolos después a 6 meses, y finalmente a intervalos anuales, dependiendo de la respuesta del tumor y de la presencia de complicaciones. El seguimiento es de por vida en todos los pacientes excepto en aquellos que fueron secundariamente enucleados, que a partir de dicho evento son remitidos al oftalmólogo y al médico de familia de su área sanitaria. En cada visita se realizó exploración oftalmológica básica, retinografías y ecografía ocular en el Servicio de Oftalmología y pruebas para detección de metástasis en el Servicio de Oncología Radioterápica.

\section{Métodos estadísticos: registro de datos}

Los datos basales analizados fueron edad, sexo, ojo derecho o izquierdo, agudeza visual corregida inicial, localización anteroposterior del tumor (predominantemente de cuerpo ciliar, preecuatorial, ecuatorial, postecuatorial, con fóvea incluida, yuxtafoveal, foveal no yuxtapapilar, yuxtapapilar no foveal y simultáneamente foveal y yuxtapapilar), localización meridional (superior, superotemporal, temporal, inferotemporal, inferior, inferonasal, nasal, superonasal y foveal), espesor tumoral, diámetro transversal (vertical en los de localización foveal) y diámetro longitudinal (horizontal en los de localización foveal). Denominaremos melanomas de cuerpo ciliar a los de localización predominante en cuerpo ciliar y melanomas de coroides al resto. Los datos sobre el tratamiento fueron la fecha de tratamiento (la de la extracción de los hilos de iridio-192), el número de hilos, y las dosis de radiación en el apex y en la base del tumor (a $2 \mathrm{~mm}$ de los hilos). Los datos de seguimiento registrados fueron la fecha de la muerte, su causa si era conocida, las fechas de la recidiva tumoral (crecimiento del tumor documentado por ecografía o por oftalmoscopía), de evidencia de metástasis, de enucleación y su causa, de la última vez en que el ojo afectado conservaba una agudeza visual corregida de 0,05 o mayor y el desarrollo de las complicaciones o efectos secundarios del tratamiento más comunes, incluyendo queratoconjuntivitis seca por radiación, catarata por radiación, sinequias posteriores, glaucoma neovascular, hemovítreo, maculopatía por radiación, retinopatía por radiación con exudación subretiniana, papilopatía por radiación, dermatopatía palpebral por radiación y necrosis escleral. Se calculó el intervalo entre la fecha de tratamiento y la de detección de dichos even- 
tos. El diagnóstico de retinopatía por radiación requirió alteraciones del lecho capilar (microaneurismas, telangiectasias o isquemia capilar angiográfica), exudación retiniana, hemorragias retinianas, edema retiniano, manchas algodonosas o envainamiento vascular. Se diagnosticó maculopatía por radiación cuando se detactaba alguno de estos cambios en el área foveal. La catarata y el síndrome de ojo seco se consideraron secundarios a la radiación (y no enfermedad común intercurrente) cuando eran ipsilaterales, bien unilaterales o bien asimétricos y no existían antes del tratamiento.

\section{Métodos estadísticos: medidas de resultados, métodos de cálculo y definición de censores}

Las principales medidas de resultados en este estudio fueron las probabilidades de supervivencia causaespecífica, de supervivencia libre de enfermedad, de recidiva tumoral local, de conservación del globo ocular, de conservación de agudeza visual corregida de al menos 0,05 y de aparición de complicaciones de la braquiterapia (la estimación del porcentaje de ojos en riesgo libres de enucleación por cualquier causa y el porcentaje de ojos en riesgo que presentaron cada complicación del tratamiento). Estas probabilidades se calcularon empleando el método de Kaplan-Meier. Para calcular la supervivencia actuarial se censuraron los casos en el momento de la última visita de seguimiento, de la enucleación por cualquier causa o de la muerte por causa distinta del melanoma y se consideraron eventos las muertes por melanoma y las muertes por causa desconocida. Para calcular la supervivencia libre de enfermedad se censuraron los casos en la fecha de la última visita de seguimiento, de la enucleación por complicaciones o de la muerte sin evidencia de la enfermedad y se tomaron como eventos las recidivas tumorales, las muertes por metástasis y las muertes por causa desconocida. Para calcular la probabilidad de recidiva local se censuraron los casos en la última visita de seguimiento, la muerte o la enucleación sin evidencia de recidiva local y se contaron como eventos la evidencias de recidiva local. Para calcular la probabilidad de conservación del ojo se censuraron los casos en la última visita de seguimiento o en la muerte sin enucleación previa y contaron como eventos las enucleaciones por recidiva tumoral y las enucleaciones por complicaciones. Para calcular la probabilidad de conservación de visión útil, los casos evaluables (los que tenían una agudeza visual corregida de al menos 0,05 antes del tratamiento) se censuraron en la última visita de seguimiento o en el fallecimiento conservando agudeza visual de al menos 0,05 en el ojo afectado y se consideraron eventos la fecha de la última visita en la que conservaban una agudeza visual corregida de al menos 0,05 en el ojo que en la siguiente visita tenían agudeza visual inferior a 0,05 . Para calcular las probabilidades de complicaciones de la braquiterapia, se censuraron los casos en la fecha de la última visita de seguimiento, muerte o enucleación sin evidencia de la complicación analizada y se consideraron eventos en la fecha de la primera exploración oftalmológica que detectó tal complicación. Para calcular la probabilidad de enucleación secundaria a complicaciones de la radioterapia se censuraron los casos en la última visita de seguimiento, en la muerte sin enucleación o en el momento de la enucleación no debida a complicaciones y se contaron como eventos en la fecha de enucleación por complicaciones.

\section{Resultados}

\section{Estadísticos descriptivos: pacientes y tumores}

Se registraron 241 pacientes tratados con braquiterapia con iridio-192 por M.U.P. entre enero de 1983 y junio de 2005. Todos los casos eran unilaterales por lo que en adelante nos referiremos indistintamente a ojos o pacientes según el contexto. Había $108(45 \%)$ varones y $133(55 \%)$ mujeres y la media de edad era 55 años (entre 21 y 88). Al diagnóstico la agudeza visual corregida en el ojo afectado era de al menos 0,05 en 191 pacientes $(79 \%)$ y peor que 0,05 en $47(19,5 \%)$ pacientes. En 3 pacientes no estaba registrada la agudeza visual inicial. En 130 pacientes $(54 \%)$ estaba afectado el ojo derecho y en 111 pacientes $(46 \%)$ el ojo izquierdo. El espesor tumoral era de $1,6 \mathrm{~mm}$ a $12,2 \mathrm{~mm}(5,6 \mathrm{~mm}$ de media), la base transversal era de $5,0 \mathrm{~mm}$ a $17,0 \mathrm{~mm}(10,5$ $\mathrm{mm}$ de media) y la base longitudinal era de $5,0 \mathrm{~mm}$ a $18,8 \mathrm{~mm}$ (10,9 $\mathrm{mm}$ de media). El espesor tumoral fue inferior a $3 \mathrm{~mm}$ en 22 casos (9\%), entre $3 \mathrm{~mm}$ y $10 \mathrm{~mm}$ en 213 casos $(88,5 \%)$ y superior a $10 \mathrm{~mm}$ en 6 casos (2,5\%). La localización anteroposterior del tumor era predominantemente de cuerpo ciliar en 13 ojos (5\%), preecuatorial en 23 ojos $(9,5 \%)$, ecuato- 
rial en 58 ojos (24\%), postecuatorial en 98 ojos (41\%), con fóvea incluida en 3 ojos (1\%), yuxtafoveal en 33 ojos (14\%), foveal no yuxtapapilar en 8 ojos (3\%), yuxtapapilar no foveal en 3 ojos (1\%) y simultáneamente foveal y yuxtapapilar en 2 ojos (1\%). La localización meridional del tumor era superior en 11 ojos (5\%), superotemporal en 52 ojos (22\%), temporal en 37 ojos (15\%), inferotemporal en 46 ojos (19\%), inferior en 24 ojos (10\%), inferonasal en 23 ojos (9\%), nasal en 14 ojos (6\%), superonasal en 24 ojos (10\%) y foveal en 10 ojos (4\%). El espesor tumoral medio de los melanomas con afectación predominante de cuerpo ciliar fue de 7 $\mathrm{mm}$. mientras que el espesor tumoral medio del resto de melanomas (melanomas de coroides) fue de $5,5 \mathrm{~mm}(\mathrm{p}=0,026)$. Ningún paciente tenía metástasis clínicas en el momento del diagnóstico.

\section{Estadísticos descriptivos: tratamiento}

Se habían tratado previamente 5 ojos con termoterapia transpupilar. El resto no habían recibido tratamiento previo. El número de hilos radioactivos empleados en cada ojo varió de 2 a 5 . La dosis apical osciló aproximadamente entre 70 y 80 Gy y la dosis basal media fue de aproximadamente $200 \mathrm{~Gy}$.

\section{Seguimiento}

Se perdió el seguimiento en 41 pacientes (17\%). El seguimiento fue entre 0 meses (por incluir a los últimos pacientes tratados, que aún no habían tenido su primera visita de seguimiento) y 20 años (media de 5,4 años y mediana de 4,3 años).

\section{Resultados}

Las probabilidades actuariales de supervivencia libre de enfermedad a 5 años y a 10 años fueron del $77 \%$ (intervalo de confianza (I.C.) del 95\%: 72, 83) y del 64\% (I.C. del 95\%: 56, 73) respectivamente (Figura 1). Se conoció la muerte de 28 pacientes, 14 por melanoma, 7 por causas desconocidas y 7 por causas ajenas al melanoma. Las probabilidades actuariales de supervivencia causa-específica a 5 y a 10 años fueron del 92\% (I.C. del 95\%: 88, 96) y del $86 \%$ (I.C. del 95\%: 80, 92), respectivamente (Figura 1). En 38 pacientes hubo recidiva local. Las probabi-

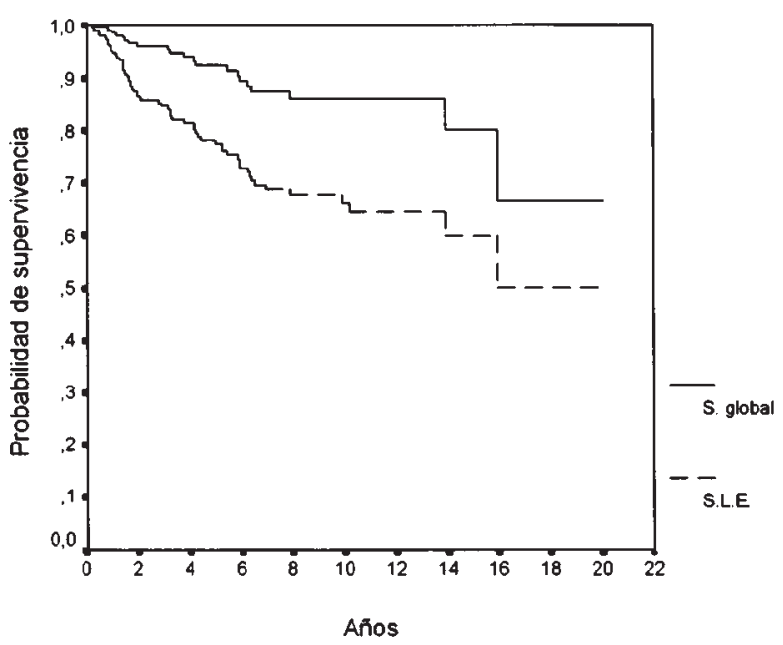

Figura 1. Curvas de Kaplan-Meier que muestran la probabilidad de supervivencia global, para la que se consideraron eventos el fallecimiento por metástasis y el fallecimiento por causa desconocida, y la probabilidad de supervivencia libre de enfermedad (S.L.E.), para la que se consideraron eventos la recidiva local, el fallecimiento por metástasis y el fallecimiento por causa desconocida.

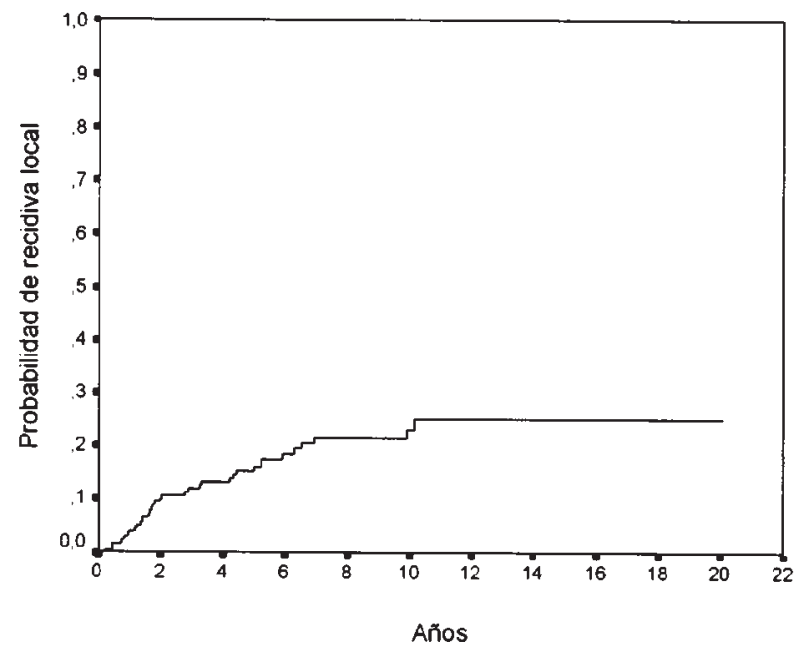

Figura 2. Curva de Kaplan-Meier inversa que muestra la probabilidad de recidiva local del tumor.

lidades actuariales de recidiva local a 5 años y a 10 años fueron del 16\% (I.C. del 95\%: 11, 21) y del 25\% (I.C. del 95\%: 18, 33) respectivamente (Figura 2). De los casos con recidiva tumoral local, un paciente se trató secundariamente con termoterapia transpupilar y otro continuó su asistencia en otro centro, el resto se trataron con enucleación. Se enuclearon secundariamente 64 ojos $(26 \%), 36(15 \%)$ 


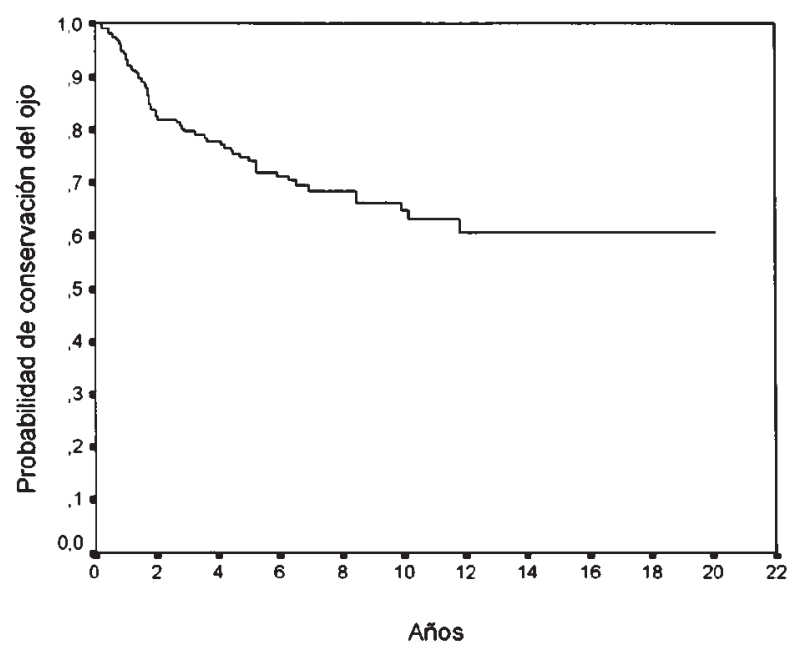

Figura 3. Curva de Kaplan-Meier que muestra la probabilidad de conservación del globo ocular, para la que se consideraron eventos la enucleación por recidiva y la enucleación por complicaciones.

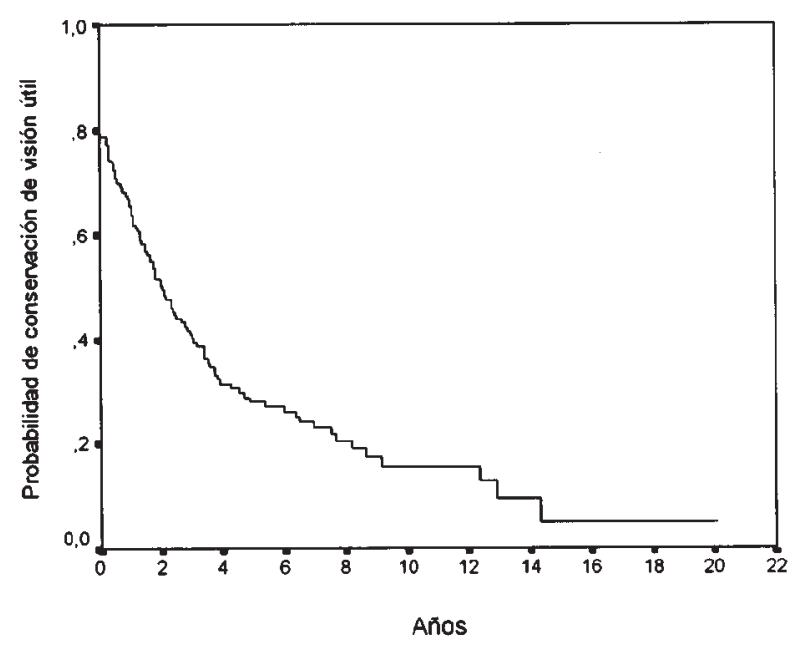

Figura 4. Curva de Kaplan-Meier que muestra la probabilidad de conservación de visión útil, definida como la agudeza visual de al menos 0,05 .

por recidiva local del tumor y $28(11 \%)$ por complicaciones del tratamiento. En dos pacientes se desconocen las fechas de enucleación. Las probabilidades de conservar el ojo a 5 años y a 10 años fueron del 74\% (I.C. del 95\%: 68, 80) y del 63\% (I.C. del 95\%: 55, 71) respectivamente (Figura 3). De los 191 ojos que tenían una agudeza visual corregida basal de al menos $0,05,188$ tenían datos de seguimiento completos en cuanto a agudeza visual. Estos pacientes se consideraron evaluables para el análisis conservación

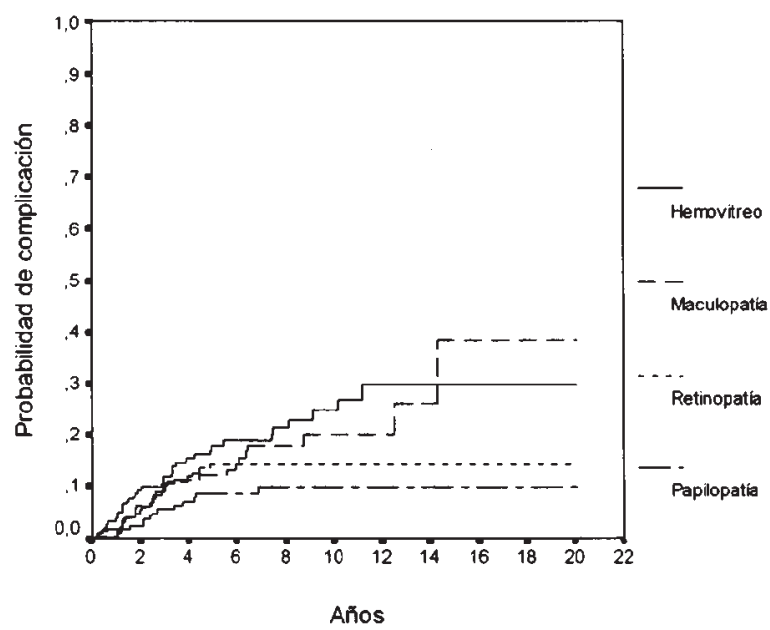

Figura 5. Curvas de Kaplan-Meier inversa que muestran las probabilidades de hemovítreo, maculopatía por radiación, retinopatía por radiación con exudación subretiniana y neuropatía óptica por radiación.

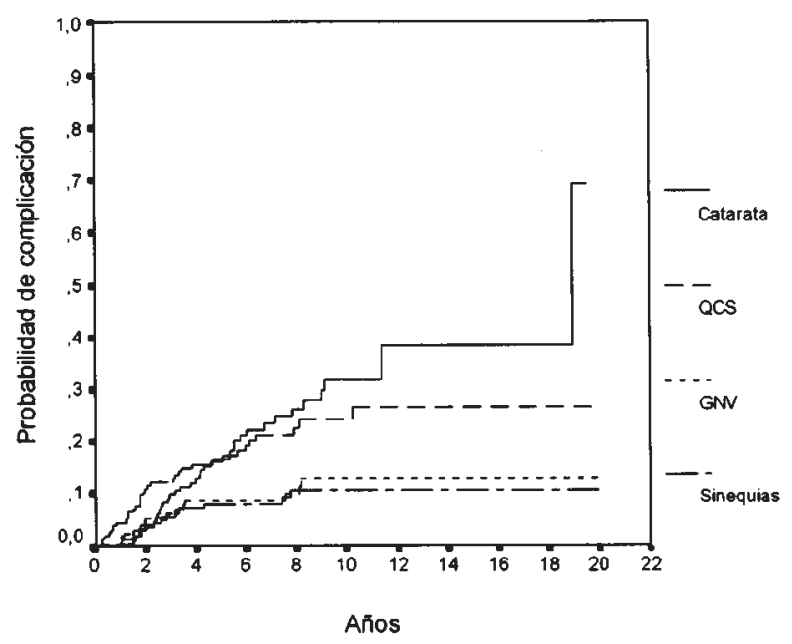

Figura 6. Curvas de Kaplan-Meier inversa que muestran las probabilidades de catarata por radiación, queratoconjuntivitis seca por radiación (QCS), glaucoma neovascular (GNV) y sinequias posteriores.

de agudeza visual. Las estimaciones de KaplanMeier de los porcentajes de ojos que conservan una agudeza visual de al menos 0,05 a 1,5 y 10 años fueron del 64\% (I.C. del 95\%: 57, 71), del 28\% (I.C. del 95\%: 21,35 ) y del 15\% (I.C. del 95\%: 8, 23) respectivamente (Figura 4). Las Figuras 5 y 6 muestran las curvas de Kaplan-Meier para el riesgo de desarrollar las complicaciones más frecuentes. Las probabilidades a 5 años de desarrollar complicaciones en los 
ojos en riesgo fueron: hemovítreo, $18 \%$ (I.C. del 95\%: 12, 24); catarata por radiación, $18 \%$ (I.C. del $95 \%, 12 ; 25)$; queratoconjuntivitis sicca por radiación, 16\% (I.C. del 95\%: 10, 21); retinopatía por radiación con exudación subretiniana, 14\% (I.C. del 95\%: 9, 20); maculopatía por radiación, 13\% (I.C. del 95\%: 8, 18); papilopatía por radiación, 9\% (I.C. del 95\%: 4, 13); glaucoma neovascular, $8 \%$ (I.C. del 95\%: 4,12 ); sinequias posteriores, $8 \%$ (I.C. del 95\%: $4,12)$; dermatopatía palpebral por radiación, $5 \%$ (I.C. del 95\%: 2, 8) y necrosis escleral, 5\% (I.C. del 95\%: 1, 8). El riesgo de dermopatía y de necrosis escleral fue significativamente mayor en pacientes con melanomas de cuerpo ciliar. La probabilidad a 5 años de dermopatía fue del 54\% (I.C. del 95\%: 27, 81) en ojos con melanoma de cuerpo ciliar y del $2 \%$ (I.C. del 95\%: 0,4) en ojos con melanoma de coroides ( $\mathrm{p}$ $<0.001)$. La probabilidad a 5 años de necrosis escleral fue del 64\% (I.C. del 95\%: 32, 95) en ojos con melanoma de cuerpo ciliar y del 1\% (I.C. del 95\%: 0, 3 ) en ojos con melanoma de coroides ( $p<0.001)$. La incidencia de queratoconjuntivitis seca en ojos con tumor de localización temporal o superotemporal fue del $29 \%$, mientras que en el resto de localizaciones fue del $8 \%$ ( $p<0,001)$. Se enuclearon 28 ojos por complicaciones de la braquiterapia. Las causas de enucleación secundaria por complicaciones fueron glaucoma neovascular en 12 casos, necrosis escleral en 4 casos, glaucoma neovascular y necrosis escleral simultáneamente en 1 caso, desprendimiento exudativo total de retina en 2 casos, phthisis bulbi en 5 casos, hemovítreo masivo en un caso y retinopatía por radiación con exudación subretiniana masiva en un caso. Dos casos fueron enucleados en su hospital local de procedencia por complicaciones no documentadas. La probabilidad a 5 años de enucleación por complicaciones fue del 13\% (I.C. del 95\%: 8, 18). La probabilidad de enucleación por complicaciones en ojos con melanomas de cuerpo ciliar fue significativamente mayor que en ojos con melanoma de coroides. Las estimaciones a 5 años fueron del $58 \%$ (I.C. del 95\%: 27, 89) y del 11\% (I.C. del 95\%: 6, 16) respectivamente $(\mathrm{p}<0.001)$.

\section{Discusión}

Durante las dos últimas décadas la braquiterapia se ha utilizado frecuentemente como tratamiento que permitiera conservar el ojo y la función visual en pacientes seleccionados con M.U.P., basándose en los resultados de supervivencia a corto plazo observados en varias series. Sin embargo la controversia sobre el tratamiento más adecuado de estos pacientes se ha prolongado durante estos años y algunos autores temían que el tratamiento conservador pudiera incrementar el riesgo de metástasis. El C.O.M.S. desarrolló un ensayo clínico multicéntrico, controlado y aleatorizado para responder a esta cuestión. Este ensayo comparó los resultados de la técnica de braquiterapia con placas de iodo- 125 con la enucleación en pacientes con melanomas de coroides de tamaño mediano y evidenció tasas de supervivencia equivalentes para ambos brazos de tratamiento. En consecuencia, la técnica de braquiterapia evaluada por el C.O.M.S. puede considerarse el tratamiento estándar en pacientes con melanoma de coroides mediano ${ }^{13}$. Sin embargo existen otros radioisótopos que se han utilizado o se utilizan en la braquiterapia de los M.U.P. Se considera el iodo-125 como el isótopo estándar en la braquiterapia del melanoma de coroides, debido a su baja energía media, de $0,039 \mathrm{MeV}$, a su fácil escudamiento $(0,01 \mathrm{~cm}$. de plomo reduce la exposición al $10 \%$ ) y a la disponibilidad comercial de fuentes bien empaquetadas, calibradas y con control de calidad. El cobalto-60 cayó en desuso debido a que su alta energía (de 1,77 a $1,33 \mathrm{MeV}$ ) hacía imposible escudar los tejidos sanos adyacentes $(4,6 \mathrm{~cm}$. de plomo reducen la exposición al 10\%). El paladio-103 aporta mayor reducción de dosis, mejorando la radioprotección del personal respecto al yodo- $125^{20}$. La energía media del iridio192 , de $0,38 \mathrm{MeV}$ es relativamente baja y por lo tanto es aceptable en cuanto a radioprotección. Sin embargo es 10 veces mayor que la del yodo- 125 y requiere $1,2 \mathrm{~cm}$ de plomo para reducir la exposición al $10 \%$, lo que impide proteger los tejidos orbitarios (glándula lagrimal) y multiplica la exposición del personal sanitario respecto a la que se produce con el yodo- $125^{21,22}$. La vida media del iridio-192, de 74,2 días, se considera corta, pero es larga si la comparamos con la media de la duración del tratamiento, en torno a 6 días, de modo que su actividad se reduce sólo en un pequeño porcentaje durante el tratamiento ${ }^{18}$. Con el rutenio-106, que a diferencia de los anteriores es emisor beta, y cuya energía media es de 3,54 MeV, el espesor de los tumores tratables está limitado a menos de $5 \mathrm{~mm}$, lo que no permite utili- 
zarlo en gran parte de los casos candidatos a braquiterapia ${ }^{23}$. Sin embargo es fácil de escudar $(0,07 \mathrm{~cm}$ de plomo reducen la exposición del rutenio al 10\%) ${ }^{4}$. En 1983 en los Servicios de Oftalmología y Oncología Radioterápica de la Cínica Puerta de Hierro se desarrolló una técnica de braquiterapia con hilos de iridio-192 en melanomas de coroides. El uso de la braquiterapia se basó en los datos existentes de otras instituciones que habían publicado tasas de supervivencia en pacientes tratados con braquiterapia con placas de cobalto-60, rutenio 106 y yodo-125, que parecían comparables con las tasas publicadas para pacientes enucleados ${ }^{24-26}$. La razón de utilizar hilos de iridio-192 fue la disponibilidad del iridio192 y de los materiales requeridos por la técnica, así como el bajo coste de este radioisótopo. En pacientes con tumores malignos, los tratamientos que conservan el órgano deben conseguir tasas de supervivencia comparables con otros tratamientos. Aunque los resultados de los estudios retrospectivos pueden estar sesgados por la selección de casos y por otros factores no controlados, las tasas de supervivencia del presente estudio no son demasiado diferentes de otras series y ensayos. En el C.O.M.S. las tasas de supervivencia a 5 años fueron de poco más del $80 \%$ para ambos grupos ${ }^{13}$. Nuestro estudio tiene además las siguientes limitaciones. En primer lugar, no se continuó sistemáticamente el seguimiento de los pacientes después de la enucleación. Esto se hizo por considerar la enucleación secundaria una intervención susceptible per se de modificar los resultados de supervivencia, de modo que a partir de dicho evento no podría atribuirse la supervivencia o el fallecimiento exclusivamente a la braquiterapia con iridio-192, que es la intervención que se pretendía evaluar. Se ha demostrado la asociación entre la recidiva local y riesgo de metástasis ${ }^{27}$ por lo que la supervivencia causa-específica de nuestro estudio puede estar sobreestimada, aunque ello no altere la supervivencia libre de enfermedad. Por otra parte, la mayoría de los pacientes de nuestra serie residen en otras áreas sanitarias o comunidades autónomas y la mayoría de los decesos suceden fuera de nuestro hospital, de modo que a menudo las causas documentadas de la muerte no son accesibles. En la mayoría de los casos el hecho del fallecimiento y su fecha se conocen mediante llamada telefónica al domicilio del paciente y la información sobre la causa de la muerte es la que dan sus familiares. Esto es importante teniendo en cuenta que la edad media al diagnóstico en melanoma de coroides es mayor que en otros cánceres y, por lo tanto el riesgo de muerte por otras causas es mayor. Entre uno de cada cuatro y el $40 \%$ de las muertes en los pacientes con melanoma de coroides tratados con braquiterapia en otras series se deben a enfermedades intercurrentes y no al melanoma de coroides $^{13,28}$. Al contar la muerte por causa desconocida como evento para determinar las supervivencias en nuestro estudio, es probable que estemos subestimandolas. Secundariamente al aumento de la supervivencia, el objetivo de la braquiterapia es la conservación de la función visual (la máxima función visual posible). La prueba clínica más utilizada para valorar la función visual es la agudeza visual corregida (la mejor agudeza visual posible con ayuda óptica). De los 556 ojos tratados con braquiterapia en el C.O.M.S. que tenían previamente agudezas visuales de más de 0,1 , el $43 \%$, tenían a los 3 años del tratamiento agudezas visuales de 0,1 o peor $^{29}$. En un estudio retrospectivo del grupo del Wills Eye Hospital, de 1106 pacientes con agudezas visuales de más de 0,1 , tenían agudezas visuales finales de 0,1 o peor, el $34 \%$ a 5 años, el $68 \%$ a 10 años y el $87 \%$ a 15 años $^{30}$. En nuestra serie los resultados de conservación de agudeza visual son aparentemente peores. Otro objetivo relevante de los tratamientos conservadores es la conservación de la integridad corporal. Los pacientes a menudo expresan su preocupación ante la posibilidad de someterse a enucleación incluso tras informarles de que la probabilidad de conservar una cantidad de visión útil en el ojo afectado es muy baja. En nuestra serie la tasa de enucleaciones debidas a complicaciones por radioterapia es mayor que en el C.O.M.S. La proporción de pacientes tratados con braquiterapia del C.O.M.S. que fueron sometidos a enucleación secundaria fue del $12 \%$ a 5 años. La causa más frecuente fue el fracaso local del tratamiento (10\%). En nuestra serie la probabilidad de conservación anatómica del ojo es notablemente menor, aunque los datos no son directamente comparables porque nosotros hemos incluido melanomas de cuerpo ciliar, en los que hemos demostrado mayor probabilidad de terminar en enucleación por complicaciones de la braquiterapia, y que fueron excluidos en el C.O.M.S ${ }^{12}$. Otros autores han publicado pequeños subgrupos de pacientes tratados con iridio-192 $2^{31-33}$ En su serie de 85 pacientes, con 30 de ellos tratados 
con iridio-192, Petrovich et al. atribuían una reducción de la tasa de enucleación principalmente al cambio del iridio-192 por el yodo-125 $5^{34}$. En una serie de 329 pacientes, 58 de ellos tratados con iridio192, Gündüz et al consideraron que el radioisótopo iridio-192 es un factor de riesgo de complicaciones por radiación y de resultados visuales pobres versus yodo- $125^{35}$. Otros estudios retrospectivos confirman peor resultado visual y mayor incidencia de enucleación secundaria con iridio- $192^{30}$. Gündüz et al., en una serie de melanomas de cuerpo ciliar tratados con braquiterapia con iodo-125, rutenio-106, cobalto-60 e iridio-192 publicaron buenos resultados en cuanto a conservación del ojo, con un $6 \%$ de enucleaciones por complicaciones ${ }^{36}$. Requieren comentario aparte ciertas complicaciones de nuestra serie, poco frecuentes con otros radioisótopos. La queratoconjuntivitis seca por radiación y la dermopatía por radiación en los párpados son complicaciones descritas en ojos con melanoma de cuerpo ciliar tras el tratamiento mediante irradiación con partículas cargadas ${ }^{37}$ pero no descritas como frecuentes tras la braquiterapia con placas ${ }^{38}$, incluyendo series de pacientes con melanoma de cuerpo ciliar $^{36}$. La imposibilidad de escudamiento efectivo en los hilos de iridio192 extraesclerales puede explicar la alta incidencia de complicaciones que afectan a los tejidos perioculares (dermopatía y síndrome de ojo seco) en los pacientes de nuestra serie. La elevada incidencia de necrosis escleral en el subgrupo de melanomas de cuerpo ciliar de nuestra serie podría explicarse también por la falta de escudamiento que proteja la conjuntiva, con las consecuencias de necrosis conjuntival y pérdida del efecto protector que la conjuntiva sana pueda tener sobre la esclera isquémica. La alta energía del iridio-192 también podría haber contribuido a la incidencia mayor de enucleaciones por complicaciones. En conclusión, la braquiterapia con hilos epiesclerales de iridio-192 se demuestra efectiva en el tratamiento del M.U.P. atendiendo a los resultados prioritarios de supervivencia. Sin embargo los resultados en cuanto a la conservación del ojo y la conservación de función visual son menos favorables que en la braquiterapia con yodo-125. Nosotros dejamos de indicar esta técnica en los melanomas de cuerpo ciliar en 1995, cuando detectamos una elevada incidencia de complicaciones graves de la braquiterapia con iridio-192 en estos casos, que acababan con demasiada frecuencia en enucleación. La justificación actual de esta técnica frente a las placas de yodo-125 en el tratamiento de los melanomas de coroides sólo puede argumentarse desde un punto de vista económico en el contexto de una financiación restrictiva.

\section{Agradecimientos}

A la Dra. Isabel Millán, del Servicio de Bioestadística del Hospital Universitario Puerta de Hierro, que realizó el análisis estadístico.

\author{
Correspondencia: \\ Dr. S. Valverde Almohalla \\ C/ Emiliano Barral, 13-A. Escalera derecha, $6^{\circ} \mathrm{B}$ \\ E-28043 Madrid
}

\section{Bibliografía}

1. Dorn HF, Cutler SJ. Morbidity from cancer in the United States (USPHS Monograph 56). Washington DC, US Government Printing Office, 1959.

2. Jensen OA. Malignant melanomas of the uvea in Denmark, 1943-1952. A clinical, histopathological, and prognostic study. Acta Ophthalmol. Suppl. 1963; 75:1.

3. Collaborative Ocular Melanoma Study Group. The Collaborative Ocular Melanoma Study (COMS) randomized trial of pre-enucleation radiation of large choroidal melanoma II: initial mortality findings. COMS repor no. 10. Am J Ophthalmol 1998;125:779-796.

4. Robertson DM, Earle J, Kline RW. Brachytherapy for choroidal melanoma. In: Ryan SJ, Schachat AP, eds. Retina.3rd ed. St. Louis, Mo: MosbyInc;2001:739-752.

5. The Collaborative Ocular Melanoma Study Group. Mortality in patients with small choroidal melanoma. COMS report no.4. Arch Ophthalmol 1997;115:886-893.

6. Shields CL, Cater J, Shields JA, Sinh AD, Santos MCM, Carvalho C. Combination of clinical factors predictive of growth of small choroidal melanocytic tumors. Arch Ophthalmol 2000;118:360-364.

7. Char DH, Stone RD, Irvine AR, Crawford JB, Hilton GF, Lonn LI et al. Diagnostic modalities in choroidal melanoma. Am J Ophthalmol 1980;89:223-230.

8. Albert DM. The ocular melanoma story. LIII Edward Jackson Memorial Lecture: part II. Am J Ophthalmol 1997;123:729-741.

9. Zimmerman LE, McLean IW, Foster WD. Does enucleation of the eye containing a malignant melanoma prevent or accelerate the dissemination of tumour cells. Br J Ophthalmol 1978;62:420-425. 
10. Manschot W, van Pepperzeel JA. Choroidal melanoma enucleation or observation? A new approach. Arch Ophthalmol 1980;98:71-77.

11. Singh AD, Rennie IG, Kivela T, Seregard S, Grossniklaus H. The Zimmerman-McLean-Foster hypothesis: 25 years later. Br J Ophthalmol 2004;88:962-967.

12. The Collaborative Ocular Melanoma Study Group. The COMS randomized trial of iodine 125 brachytherapy for choroidal melanoma, II: characteristics of patients enrolled and not enrolled. COMS report no.17. Arch Ophthalmol 2001;119:951-965

13. The Collaborative Ocular Melanoma Study Group. The COMS randomized trial of iodine 125 brachytherapy for choroidal melanoma, III: initial mortality findings. COMS report no.18. Arch Ophthalmol 2001;119:969-982.

14. Sieving PA. Fifteen years of work. The COMS outcomes for medium-sized choroidal melanoma. Arch Ophthalmol 2001;119:1067-1068.

15. Gordon KB, Char DH, Sagerman RH. Late effects of radiation on the eye and ocular adnexa. Int J Radiation Oncology Biol Phys.1995;31:1123-1139.

16. Earle J, Kline RW, Robertson DM. Selection of 125I for the Collaborative Ocular Melanoma Study. Arch Ophthalmol 1987;105:763-764.

17. Encinas JL, Bañuelos J, Saquetti A. Tratamiento conservador del melanoma de coroides con iridio-192. Studium Ophthalmologicum 1987;6:37-4714.

18. Valcárcel F, Valverde S, Cárdenes H, Cajigal C, de la Torre A, Magallón R et al. Episcleral Iridium-192 wire therapy for choroidal melanomas. Int J Radiation Oncology Biol Phys 1994; 30: 1091-1097.

19. Valverde Almohalla S, Jiménez Vales F, Arteaga Sánchez A, Hijós Gastón M, Cajigal Morales C, Encinas Martín JL. Verificación ecográfica intraoperatoria de la posición de hilos de Iridio 192 en melanoma de coroides. Arch Soc Esp Oftalmol 1997; 72: 191-196.

20. Finger PT. Berson A, Szechter A. Palladium-103 plaque radiotherapy for choroidal melanoma. Results of a 7-year study. Ophthalmology 1999;106:606-613.

21. Laube T, Flühs D, Kessler C, Bornfeld N. Determination of surgeon's absorbed dose of iodine-125 and ruthenium-106 ophthalmic plaque surgery. Ophthalmology 2000;107:366369 .

22. Escalada C, Cutanda F, Pifarré X, Valcárcel F, Valverde S, Paredes MC. Dosis en manos de profesionales que realizan implantes oculares de iridio con fines terapéuticos. Radioprotección 1996; $\mathrm{n}^{\circ}$ extraordinario: 92-93.

23. Rouberol F, Roy P, Kodjikian L, Gerard JP, Jean-Louis B and Grange D. Survival, anatomic, and functional longterm results in choroidal and ciliary body melanoma after ruthenium brachitherapy (15 years' experience with betarays). Am J Ophthalmol 2004;137:893-900.
24. Stallard HB. Radiotherapy for malignant melanoma of the choroid. Br J Ophthalmol 1966;50:147-155.

25. Packer S, Rotman M. Radiotherapy of choroidal melanoma with iodine-125. Ophthalmology 1980;87:582-590.

26. Lommatzsch PK. B-irradiation with $106 \mathrm{Ru} / 106 \mathrm{Rh}$ applicators; 16 years' experience. Arch Ophthalmol 1983;101: 713-717.

27. Egan KM, Ryan LM; Gragoudas ES. Survival implications of enucleation after definitive radiotherapy for choroidal melanoma. An example of regression on time-dependent covariates. Arch Ophthalmol 1998;116:366-370.

28. Kroll S, Char DH, Quivey J, Castro J. A comparison of cause-specific melanoma mortality and all-cause mortality in survival analyses after radiation treatment for uveal melanoma. Ophthalmology 1998;105:2035-2045.

29. Collaborative Ocular Melanoma Study Group. Collaborative Ocular Melanoma Study (COMS) randomized trial of iodine 125 brachytherapy for medium choroidal melanoma, I. Visual acuity after 3 years. COMS report no.16. Ophthalmology 2001;108:348-366.

30. Shields CL, Shields JA, Cater J et al. Plaque radiotherapy for uveal melanoma. Long-term visual outcome in 1106 consecutive patients. Arch Ophthalmol 2000;118:1219-1228.

31. Gerard JP, Grange JD, Romestaing P, Sentenac I, De Laroche G, Montbarbon X et al. Curietherapy using a ruthenium and iridium disk in the treatment of choroid melanoma. Ann Radiol 1989;32:107-111.

32. Lean EK, Cohen DM, Liggett PE, Luxton G, Langholz B, Lau $\mathrm{R}$ et al. Episcleral radioactive plaque therapy: initial clinical experience with 56 patients. Am J Clin Oncol 1990;13:185-190.

33. Pizzi GB, Marchetti C, Zemella M, Turcato G, Stea L, Polico R et al. Brachytherapy in choroid tumors. Radiol Med1994;88:858-862.

34. Petrovich Z, Luxton G, Langholz B, Astrahan MA, Liggett PE. Episcleral plaque radiotherapy in the tratment of uveal melanomas. Int $\mathrm{J}$ Radiation Oncology Biol Phys 1992;24:247-251.

35. Gündüz K, Shields CL, Shields JA, Cater J, Freire JE, Brady LW. Radiation retinopathy following plaque radiotherapy for posterior uveal melanoma. Arch Ophthalmol 1999;117:609-614.

36. Gündüz K, Shields CL, Shields JA, Cater J, Freire JE, Brady LW. Plaque radiotherapy of uveal melanoma with predominant ciliary body involvement. Arch Ophthalmol 1999;117:170-177.

37. Gragoudas ES, Goitein M, Verhey L, et al. Proton beam irradiation of uveal melanomas: Results of a 5 1/2 year study. Arch Ophthalmol 1982;100:928-934.

38. Finger PT. Tumour location affects the incidence of cataract and retinopathy after ophthalmic plaque radiation therapy. Br J Ophthalmol 2000;84:1068-1070. 